\title{
Histopathology (2)
}

\section{Prognostic impact of tumour-stroma ratio in early-stage oral tongue cancers}

\begin{tabular}{|c|c|}
\hline Journal: & Histopathology \\
\hline Manuscript ID & HISTOP-10-17-0664.R2 \\
\hline Wiley - Manuscript type: & Original Article \\
\hline Date Submitted by the Author: & $\mathrm{n} / \mathrm{a}$ \\
\hline Complete List of Authors: & $\begin{array}{l}\text { Almangush, Alhadi; Helsingin Yliopisto Laaketieteellinen tiedekunta } \\
\text { Heikkinen, Ilkka } \\
\text { Bakhti, Nassira } \\
\text { Makinen, Laura } \\
\text { Kauppila, Joonas; University of Oulu, Dpt. of Surgery; University of Oulu, } \\
\text { Dpt. of Pathology } \\
\text { Pukkila, Matti } \\
\text { Hagström, Jaana; Haartman Institute, Department of Pathology } \\
\text { Laranne, Jussi } \\
\text { Soini, Ylermi } \\
\text { Kowalski, Luis; A.C.Camargo Cancer Hospital, Department of Head and } \\
\text { Neck Surgery and Otorhinolaryngology } \\
\text { Grénman, Reidar } \\
\text { Haglund, Caj; Helsinki University Central Hospital, Surgery; } \\
\text { Makitie, Antti } \\
\text { Coletta, Ricardo } \\
\text { Leivo, Ilmo; University of Turku, Pathology } \\
\text { Salo, Tuula; Oulu Center for Cell-Matrix Research, University of Oulu, Oulu, } \\
\text { Finland, Institute of Dentistry; University of Helsinki, Helsinki, Finland, } \\
\text { Institute of Dentistry }\end{array}$ \\
\hline Keywords: & Oral tongue cancer, prognosis, marker, survival, tumour-stroma ratio \\
\hline
\end{tabular}

\section{SCHOLARONE ${ }^{\text {m }}$ \\ Manuscripts}


1 Prognostic impact of tumour-stroma ratio in early-stage oral tongue

\title{
cancers
}

\author{
Running title: Tumour-stroma ratio in oral tongue cancers
}

\footnotetext{
${ }^{1}$ Department of Pathology, University of Helsinki, Helsinki, Finland

${ }^{2}$ Department of Pathology, University of Turku, Turku, Finland

${ }^{3}$ Institute of Dentistry, University of Misurata, Misurata, Libya

${ }^{4}$ Department of Oral and Maxillofacial Diseases, University of Helsinki, Helsinki, Finland

${ }^{5}$ Master of Biostatistics, Department of statistics, Faculty of sciences, Hasselt University, Belgium.

${ }^{6}$ Department of Otorhinolaryngology - Head and Neck Surgery, Helsinki University Hospital and University of Helsinki, Helsinki, Finland

${ }^{7}$ Cancer and Translational Medicine Research Unit, Medical Research Center Oulu, University of Oulu and Oulu University Hospital, Oulu, Finland

${ }^{8}$ Department of Molecular Medicine and Surgery, Karolinska Institutet, Karolinska University Hospital, Stockholm, Sweden

${ }^{9}$ Department of Otorhinolaryngology and Head and Neck Surgery, Kuopio University Hospital, Kuopio Finland

${ }^{10}$ HUSLAB, Department of Pathology, Helsinki University Hospital, University of Helsinki, Helsinki, Finland

${ }^{11}$ Research Programs Unit, Translational Cancer Biology, University of Helsinki, Helsinki, Finland

${ }^{12}$ Department of Otorhinolaryngology and Head and Neck Surgery, Tampere University Hospital, Tampere Finland

${ }^{13}$ Department of Pathology and Forensic Medicine, University of Eastern Finland, Kuopio, Finland

${ }^{14}$ Department of Head and Neck Surgery and Otorhinolaryngology, A.C. Camargo Cancer Center, São Paulo-SP, Brazil

${ }^{15}$ Department of Otorhinolaryngology - Head and Neck Surgery, Turku University Hospital, University of Turku, Turku, Finland

${ }^{16}$ Department of Surgery, University of Helsinki and Helsinki University Hospital, Helsinki, Finland

${ }^{17}$ Division of Ear, Nose and Throat Diseases, Department of Clinical Sciences, Intervention and Technology, Karolinska Institutet, Karolinska University Hospital, Stockholm, Sweden.

${ }^{18}$ Department of Oral Diagnosis, School of Dentistry, State University of Campinas, Piracicaba, São Paulo, Brazil

* These two authors have contributed equally to this study.
}

Corresponding author: Alhadi Almangush, DDS, $\mathrm{PhD}$

Department of Pathology, University of Helsinki, Helsinki, Finland

Telephone number: 00358452044668

e-mail: alhadi.almangush@helsinki.fi (or: alhadi.almangush@gmail.com)

Conflict of interest: The authors declare no conflict of interest.

Word count: 2184 


\section{Abstract}

2 Aims: Oral tongue squamous cell carcinoma (OTSCC) has a relatively poor outcome and there is a

3 need to identify better prognostic factors. Recently, tumour-stroma ratio (TSR) has been associated

4 with prognosis in several cancers. This multi-institutional study evaluates the prognostic value of

5 TSR from original HE-stained tumor-resection slides in a series of early-stage (cT1-2N0) OTSCC

6 patients.

7 Methods and results: A TSR cutoff value of 50\% was used to divide the patients into stroma-rich

$8 \quad(\geq 50 \%)$ and stroma-poor $(<50 \%)$ groups. The relationships between TSR and clinicopathologic

9 characteristics of 311 early-stage OTSCC cases were analyzed. The prognostic value of TSR in

OTSCC was calculated separately and in combination with a previously published cancer cell budding and depth of invasion (BD) prognostic model. A total of 89 cases (28.6\%) belonged to the stroma-rich group. In a multivariate analysis, the stroma-rich group had worse disease-free survival with a hazard ratio $(\mathrm{HR})$ of $1.81(95 \% \mathrm{CI} 1.17-2.79, P=0.008)$ and higher cancer-related mortality (HR 1.71, 95\%CI 1.02-2.86, $P=0.03$ ). The combination of the highest risk parameter scores of the TSR and the BD model showed a significant correlation with recurrence rate (HR 3.42, 95\%CI $1.71-6.82, P=0.004)$ and cancer-related mortality (HR 11.63, 95\%CI 3.83-35.31, $P<0.001)$.

Conclusions: We conclude that TSR is a simple histopathologic feature useful for prognostication of early-stage OTSCC, and suggest that TSR analyses in association with BD score could be included in routine clinical pathology reports for HE-stained slides.

Key words: Oral tongue cancer, prognosis, marker, survival, tumour-stroma ratio. 


\section{Introduction}

2 Oral tongue squamous cell carcinoma (OTSCC), the most common and aggressive cancer of the

3 oral cavity, is characterized by an unpredictable prognosis even at the early stage of the disease. The

4 incidence of OTSCC has increased worldwide even in countries where tobacco use, the major risk

5 factor for oral cancer, had decreased ${ }^{1}$. Overall, OTSCC is associated with a high risk of occult

6 lymph node metastasis, recurrence and cancer-related mortality ${ }^{2,3}$.

Treatment planning for OTSCC is generally based on disease staging, i.e. clinical assessment of the tumour, lymph nodes and distant metastases (cTNM staging). However, this staging system often fails to provide an accurate prediction for early-stage OTSCC $^{3,4}$. Therefore, there is a need for reliable and simple prognostic markers that could better identify early-stage OTSCC with an aggressive behavior. When considering conventional haematoxylin and eosin (HE) staining, previous histopathologic prognostic markers have mainly been related to cancer cells (e.g. degree of differentiation, pattern of invasion, depth of invasion, nuclear pleomorphism and mitotic activity). However, tumour growth has been shown to depend also on tumour surrounding stroma, i.e. tumour microenvironment (TME), which has an important role in cancer progression ${ }^{5}$. For individualized cancer management, it is therefore important to characterize both tumour cells and stroma.

Tumour-stroma ratio (TSR) has been identified recently as a useful prognostic tool in several solid tumours, including nasopharyngeal ${ }^{6}$, oesophageal ${ }^{7}$ and $\operatorname{colon}^{8}$ cancers. Evaluation of TSR in tissue sections is simple, quick, and reproducible $\mathrm{e}^{7,9}$. To the best of our knowledge, the prognostic significance of TSR in early-stage OTSCC has not been studied. Here, we evaluated TSR and its relationship with tumour characteristics in a multicenter cohort of early-stage OTSCC. Furthermore, we combined TSR with our previous budding and depth of invasion (BD) model ${ }^{10,11}$ to assess whether these three easily measured parameters from HE-stained sections could together provide a valuable estimate for early-stage OTSCC prognostication. 


\section{Material and Methods}

3 The cohort of this study includes 224 cases of early-stage OTSCC treated by surgical resection in

4 the five Finnish University Hospitals (Helsinki, Turku, Tampere, Oulu and Kuopio) and 87 cases

5 treated at the A.C. Camargo Cancer Center, São Paulo, Brazil ${ }^{10}$. All cases were staged according to 6 the existing 7th edition of the American Joint Committee on Cancer ${ }^{12}$. The original routine

7 histopathologic slides and follow-up data were collected for all cases. Ethics approval of the

8 institutional review boards of all participating hospitals was obtained, as was approval of the

9 Brazilian Human Research Ethics Committee and the Finnish National Supervisory Authority for

Welfare and Health (VALVIRA).

To ensure proper evaluation of the tumour-stroma ratio (TSR), training sessions were arranged and guided by an experienced head and neck pathologist (IL). Two researchers (AA \& IH) blinded to patient data scored the cases independently. In cases of disagreement, a review session was arranged. We scanned the whole tumour under low magnification $(\times 4)$ to select a field with the highest amount of stroma, and at the same time, with cancer cells at all four sides of the field ${ }^{13}$. As noted previously, areas rich in stroma were usually located near the site of deepest invasion ${ }^{7}$. Under higher magnification $(\times 10)$, the selected field was scored as stroma-poor $(<50 \%)$ or stroma-rich ( $\geq 50 \%$ ) (Fig. 1). In case of a heterogenic tumour (i.e. the tumour has both stroma-poor and stromarich areas), stroma-rich areas were selected and deemed decisive, as previously described ${ }^{7,14}$. Areas of muscle invasion and necrosis were visually excluded.

The prognostic value of TSR was first separately analyzed. Additionally, risk scores were constructed by combining the TSR and the BD model. The BD model parameters for these same samples were previously analyzed and published ${ }^{10}$. TSR and BD combination scores were set as follows: Score 0 (low-risk): all three markers (tumour budding, depth of invasion and TSR) were below the cutoff points (i.e. $<5$ buds, $<4 \mathrm{~mm}$ depth, and $<50 \%$ stroma). Score 1 (intermediate-risk): 
1 only one of the markers exceeded the cutoff point. Score 2 (high-risk): two of the markers exceeded the cutoff point. Score 3 (extremely high-risk): all markers exceeded the cutoff point.

The histologic risk score has been evaluated as previously described by BrandweinGensler $^{15,16}$. Cases with score 0 were considered to be at low risk; cases having score 1 or 2 were considered to be at intermediate risk; and cases of score 3 or more were at high risk ${ }^{16}$.

Survival analyses were conducted by using SPSS Statistics (version 24.0). Univariate analysis and Kaplan-Meier curves were created for both disease-free survival (DFS) and diseasespecific survival (DSS). In multivariate analysis, classical prognostic factors, such as age, gender, clinical stage, WHO tumour grade, and perineural invasion (PNI), were included in the multivariate Cox regression model together with TSR or with the combined scores of TSR and the BD model. The relationship between TSR and the classical prognostic variables was also analyzed.

\section{Results}

The clinicopathologic characteristics of this multicenter cohort have been published previously ${ }^{10}$. In brief, the 311 patients comprised 165 males and 146 females; 124 cases were clinically T1N0M0 and 187 cases were T2N0M0. All patients had been treated by surgical resection. Eighty-nine cases (28.6\%) were classified as stroma-rich and 222 cases (71.4\%) as stroma-poor. In the present study, there was a concordance between the observers in classifying the tumours into stroma-poor $(<50 \%)$ or stroma-rich ( $\geq 50 \%)$ in $96 \%$ of the cases (Cohen's kappa $=0.91$ ).

The relationship between TSR and clinicopathologic characteristics is summarized in Table 1. No association was found between TSR and age, gender, cTNM stage, or WHO grade (two-sided $P>$ 0.05). TSR was associated with PNI (two-sided $P=0.04$ ).

\section{Univariate survival analysis}

Stroma-rich cases were associated with higher rates of recurrences with a hazard ratio (HR) of 1.67 and $95 \%$ confidence interval $(95 \% \mathrm{CI})$ of $1.09-2.56(P=0.02)$. In addition, stroma-rich cases had 
1 more cancer-related deaths (HR 1.69, 95\%CI 1.02-2.79, $P=042$ ). Kaplan-Meier curves (Fig. 2)

2 showed a worse prognosis (both DFS and DSS) for stroma-rich cases. The combined highest scores

3 of TSR and the BD model (Table 2) were associated with higher rates of recurrences with a HR of

$4 \quad 2.82,(95 \%$ CI 1.46-5.42, $P=0.014)$, and these cases also had more cancer-related deaths (HR 10.43,

$5 \quad 95 \%$ CI 3.51-31.01, $P<0.001)$.

Among the classical prognostic factors (age, gender, cTNM stage, WHO tumour grade and

PNI) that were included in the survival analysis (Table 2), only age of the patient was associated with DFS (HR 1.79, 95\%CI 1.14-2.79, $P=0.01$ ) and DSS (HR 1.87, 95\%CI 1.09-3.18, $P=0.02$ ). The histologic risk score of Brandwein-Gensler et al ${ }^{15,16}$ did not show a significant prognostic value for DFS (HR 0.79, 95\%CI 0.34-1.85, $P=0.59$ ) or DSS (HR 1.30, 95\% 0.40-4.23, $P=0.66$ ). The other factors (gender, cTNM stage, WHO tumour grade and PNI) showed no significant association with either DFS or DSS $(P>0.05)$.

\section{Multivariate survival analysis}

In the adjusted analysis, age, gender, cTNM stage, WHO tumour grade and PNI were included in the Cox regression model to evaluate the independence of TSR for prognostication of DFS and DSS (Table 2). Stroma-rich cases were at higher risk for recurrence (HR 1.81, 95\%CI 1.17-2.79, $P=$ 0.008 ) and also had more cancer-related mortality (HR 1.71, 95\%CI 1.02-2.86, $P=0.03$ ) than stroma-poor cases. The combined highest scores of TSR and the BD model were associated with increased recurrence (HR 3.42, 95\%CI 1.71-6.82, $P=0.004)$ and with increased risk of OTSCCrelated mortality (HR 11.63, 95\%CI 3.83-35.31, $P<0.001$ ). Age of patients remained an independent prognostic factor in multivariate analysis for DFS (HR 2.02, 95\% CI 1.27-3.23, $P=$ 0.003) and DSS (HR 2.17, 95\%CI 1.24-3.79, $P=0.007$ ). The other factors had no significant prognostic value in multivariate analysis. 


\section{Discussion}

2 Several studies have evaluated prognostic markers for OTSCC ${ }^{2,17}$, but most of these studies have

3 significant shortcomings: early and late stages are combined, cancers from different subsites of the 4 tongue (oral and base) are pooled, and patient cohorts are rather small and from a single institute.

5 Moreover, the prognostic studies have mainly focused on features related to cancer cells. The tumour-stroma ratio (TSR) has already been investigated in several solid cancers based on a recent

7 meta-analysis ${ }^{13}$, and a high proportion of stroma in cancer tissue was associated with poor clinical

8 outcome. Here, for the first time, we have evaluated the prognostic value of TSR in a large 9 international multicenter cohort of early-stage OTSCC, and found that TSR is useful for early-stage

OTSCC prognostication. The prognostic value was improved if TSR analysis was combined with the BD model ${ }^{10}$.

The 7 th edition of staging manual of the American Joint Committee on Cancer (AJCC) ${ }^{12}$ does not provide valuable prognostic power for early-stage (cT1-2N0M0) OTSCC and has therefore been widely criticized ${ }^{18}$. For this reason, the 8 th edition of the AJCC staging manual ${ }^{19}$, which was released recently, has incorporated depth of invasion in the T-category. Of note, the AJCC emphasized that depth of invasion (and not tumor thickness) should be used for T-category ${ }^{19}$. The AJCC 8th will be applied for staging of new cases from January 2018, and there is a need for further research to evaluate the prognostic value of this new staging in early OTSCC (cT1-2N0M0). At the same time, the WHO histopathologic grading system which based on the differentiation of cancer cells has also been criticized due to its low prognostic performance for early $\mathrm{OTSCC}^{3,20}$. Furthermore, a histologic risk score has been introduced by Brandwein-Gensler et al as a prognostic model for OSCC ${ }^{15,16}$. However, our previous study of a Finnish cohort ${ }^{21}$ and the current study of a Finnish and Brazilian cohort did not find prognostic usefulness for this histologic risk score in early-stage OTSCC. Similarly, other recent studies have reported low prognostic performance for the histologic risk score ${ }^{11,20,22}$. 
Such shortcomings in prognostic power may lead to inappropriate management with either

ineffective treatment or overtreatment. To date, all histopathologic features incorporated into routine clinical pathology reports are related only to cancer cells. Although the role of the stroma in cancer progression has been recognized for more than a decade ${ }^{23-25}$, its applications in interpreting cancer specimens, including OTSCC, have been very limited. In this study, we found that OTSCC patients with a stroma-rich tumour had a worse prognosis than patients with less stroma (stromapoor). Importantly, TSR showed a considerably better prognostic power than conventional cTNM staging and histopathologic WHO tumour grade (Table 2). Of note, concordance between the observers in this study was very good (96\%), and an almost similar concordance (94\%) on TSR has been reported in other cancers ${ }^{6,26}$. Also the kappa values indicates good agreement between observers in this and previous studies $6,7,9,14$.

Combination of TSR with the BD model (including tumour depth of invasion and tumour budding $^{10}$ ) showed an even greater prognostic value in multivariate analysis. In the patient with the highest score, risks of recurrence and OTSCC-related mortality were 3.4-fold and 11.6-fold higher, respectively. These highest risk patients had thus an early stage cancer with abundant stroma ( $\geq 50 \%$ ), 5 or more cancer cell buds, and invasion depth of at least $4 \mathrm{~mm}$. In such patients, a multimodality treatment, including prophylactic neck dissection and possible adjuvant radiotherapy, should be considered even though such cases are clinically diagnosed as early-stage (cT1-2N0) tumours.

Tumour-host interactions play an important role in cancer progression, where the crosstalk between tumour cells and the surrounding stroma is a continuous process ${ }^{5,27}$. Different types of immune cells are located in the tumour stroma and have key roles in tumour progression. For example, tumour-associated macrophages (TAMs) are shown to promote tumour growth, inhibit anti-tumour immune responses, and their number is positively associated with poor prognosis in $\mathrm{OSCC}^{28-30}$. Inflammatory cells play important role in cancer progression and predict the prognosis 
1 of OSCC $^{31}$. Moreover, the density of the inflammatory infiltrate in OTSCC was significantly

2 correlated with cancer-associated fibroblasts $(\mathrm{CAFs})^{27}$. Vascular endothelial growth factor (VEGF)

3 and other growth factors such as epidermal growth factor (EGF) are derived from stromal cells and

4 endothelial cells and promote tumour angiogenesis that enhances tumour growth, survival and

5 metastasis $^{13,32-34}$. In OTSCC, CAFs identified by $\alpha$-smooth muscle actin have been reported as key

6 players in tumour progression ${ }^{35}$, and they serve as an important prognostic marker in oral cancers,

7 including all stages of OTSCC ${ }^{36,37}$. In addition, stromal compounds, such as activin $\mathrm{A}$, tenascin-C

8 and fibronectin have shown prognostic power in $\mathrm{OTSCC}^{38,}{ }^{39}$. However, use of

9 immunohistochemistry for such biomarkers increases costs, and the staining results may be, depending on the antibody used and the ligand expression level, difficult to analyze objectively. The benefit of TSR is that it can be evaluated from routine HE-stained slides. Evaluation of TSR in combination with cancer invasion parameters, such as tumour budding and depth of invasion, provides prognostication superior to evaluation of each parameter separately.

In conclusion, visual analysis of TSR is useful for early-stage OTSCC prognostication. Combining TSR analysis with features of cancer cell, such as budding and depth of invasion, could assist clinicians in identifying the early-stage OTSCC patients who most likely have a poor prognosis and might benefit from more aggressive treatment. TSR and the BD model are simple to evaluate from routine diagnostic slides and do not require additional staining. The results reported here need to be validated in independent studies before these prognostic parameters are included in routine pathology reports.

Acknowledgments: The authors acknowledge the funders of this study: Finnish Dental Society, the Finnish Cancer Society, Jalmari and Rauha Ahokas Foundation, K. Albin Johanssons foundation, 
1 Finska Läkaresällskapet, Sigrid Jusélius Foundation, the Helsinki University Hospital research

2 funds, the Orion Research Foundation, and Maritza and Reino Salonen Foundation.

\section{Author Contributions}

4 Institutional Coordinators: Salo T, Grénman R, Coletta RD, Kowalski LP, Leivo I, Mäkitie AA, 5 Haglund C, Soini Y, Kauppila JH, Pukkila M, Laranne J. Study concepts and study design: 6 Almangush A, Heikkinen I, Coletta RD, Salo T, Leivo I. Data acquisition and quality control of 7 data: Mäkinen L, Laranne J, Soini Y, Kauppila JH, Pukkila M, Hagström J, Kowalski LP, Leivo I. 8 Data analysis and interpretation: Almangush A, Heikkinen I, Bakhti N, Salo T, Leivo I. 9 Manuscript preparation: Almangush A, Heikkinen I, Coletta RD. Manuscript review: Grénman

R, Mäkitie AA, Hagström J, Leivo I, Salo T, Kowalski LP. Manuscript editing: Salo T, Leivo I, Mäkitie AA, Haglund C. All authors approved the final manuscript for submission.

\section{References}

1. Korvala J, Jee K, Porkola E, et al. MicroRNA and protein profiles in invasive versus non-invasive oral tongue squamous cell carcinoma cells in vitro. Exp Cell Res. 2017;350:9-18.

2. Bello IO, Soini Y, Salo T. Prognostic evaluation of oral tongue cancer: means, markers and perspectives (I). Oral Oncol. 2010;46:630-635.

3. Almangush A, Bello IO, Coletta RD, et al. For early-stage oral tongue cancer, depth of invasion and worst pattern of invasion are the strongest pathological predictors for locoregional recurrence and mortality. Virchows Arch. 2015;467:39-46.

4. Hori Y, Kubota A, Yokose T, et al. Predictive Significance of Tumor Depth and Budding for Late Lymph Node Metastases in Patients with Clinical N0 Early Oral Tongue Carcinoma. Head Neck Pathol. 2017.

5. Pietras K, Ostman A. Hallmarks of cancer: interactions with the tumor stroma. Exp Cell Res. 2010;316:1324-1331.

6. Zhang XL, Jiang C, Zhang ZX, et al. The tumor-stroma ratio is an independent predictor for survival in nasopharyngeal cancer. Oncol Res Treat. 2014;37:480-484. 
1 7. Wang $\mathrm{K}$, Ma W, Wang J, et al. Tumor-stroma ratio is an independent predictor for survival in 2 esophageal squamous cell carcinoma. J Thorac Oncol. 2012;7:1457-1461.

3 8. Hynes SO, Coleman HG, Kelly PJ, et al. Back to the future: routine morphological assessment of the tumour microenvironment is prognostic in stage II/III colon cancer in a large population-based study. Histopathology. 2017;71:12-26.

9. Li H, Yuan SL, Han ZZ, et al. Prognostic significance of the tumor-stroma ratio in gallbladder cancer. Neoplasma. 2017;64.

10. Almangush A, Coletta RD, Bello IO, et al. A simple novel prognostic model for early stage oral tongue cancer. Int J Oral Maxillofac Surg. 2015;44:143-150.

11. Sawazaki-Calone I, Rangel A, Bueno AG, et al. The prognostic value of histopathological grading systems in oral squamous cell carcinomas. Oral Dis. 2015;21:755-761.

12. Edge SB, Byrd DR, Compton CC, et al. AJCC Cancer Staging Manual, 7th edition. New York: Springer. 2010.

13. Wu J, Liang $\mathrm{C}$, Chen $\mathrm{M}$, et al. Association between tumor-stroma ratio and prognosis in solid tumor patients: a systematic review and meta-analysis. Oncotarget. 2016;7:68954-68965.

14. Dekker TJ, van de Velde CJ, van Pelt GW, et al. Prognostic significance of the tumor-stroma ratio: validation study in node-negative premenopausal breast cancer patients from the EORTC perioperative chemotherapy (POP) trial (10854). Breast Cancer Res Treat. 2013;139:371-379.

15. Brandwein-Gensler M, Teixeira MS, Lewis CM, et al. Oral squamous cell carcinoma: histologic risk assessment, but not margin status, is strongly predictive of local disease-free and overall survival. Am J Surg Pathol. 2005;29:167-178.

16. Brandwein-Gensler M, Smith RV, Wang B, et al. Validation of the histologic risk model in a new cohort of patients with head and neck squamous cell carcinoma. Am J Surg Pathol. 2010;34:676-688.

17. Bello IO, Soini Y, Salo T. Prognostic evaluation of oral tongue cancer: means, markers and perspectives (II). Oral Oncol. 2010;46:636-643.

18. Piazza C, Montalto N, Paderno A, et al. Is it time to incorporate 'depth of infiltration' in the T staging of oral tongue and floor of mouth cancer? Curr Opin Otolaryngol Head Neck Surg. 2014;22:81-89. 
1 19. Amin MB, Edge S, Greene F, et al. AJCC Cancer Staging Manual. 8th ed. New York: Springer. 2017.

3 20. Rodrigues PC, Miguel MC, Bagordakis E, et al. Clinicopathological prognostic factors of oral 4 tongue squamous cell carcinoma: a retrospective study of 202 cases. Int J Oral Maxillofac Surg. $5 \quad 2014 ; 43: 795-801$.

6 21. Almangush A, Bello IO, Keski-Santti H, et al. Depth of invasion, tumor budding, and worst pattern 7 of invasion: prognostic indicators in early-stage oral tongue cancer. Head Neck. 2014;36:811-818.

8 22. Strieder L, Coutinho-Camillo CM, Costa V, et al. Comparative analysis of three histologic grading 9 methods for squamous cell carcinoma of the lip. Oral Dis. 2017;23:120-125. 23. Sund M, Kalluri R. Tumor stroma derived biomarkers in cancer. Cancer Metastasis Rev. $11 \quad 2009 ; 28: 177-183$.

12 24. Tarin D. Role of the host stroma in cancer and its therapeutic significance. Cancer Metastasis Rev. $13 \quad 2013 ; 32: 553-566$.

25. Ueno $\mathrm{H}$, Konishi $\mathrm{T}$, Ishikawa $\mathrm{Y}$, et al. Histologic categorization of fibrotic cancer stroma in the primary tumor is an independent prognostic index in resectable colorectal liver metastasis. Am J Surg Pathol. 2014;38:1380-1386.

26. Vangangelt KMH, van Pelt GW, Engels CC, et al. Prognostic value of tumor-stroma ratio combined with the immune status of tumors in invasive breast carcinoma. Breast Cancer Res Treat. 2017.

27. Dayan D, Salo T, Salo S, et al. Molecular crosstalk between cancer cells and tumor microenvironment components suggests potential targets for new therapeutic approaches in mobile tongue cancer. Cancer Med. 2012;1:128-140.

28. Fujii N, Shomori K, Shiomi T, et al. Cancer-associated fibroblasts and CD163-positive macrophages in oral squamous cell carcinoma: their clinicopathological and prognostic significance. J Oral Pathol Med. 2012;41:444-451.

29. Alves AM, Diel LF, Lamers ML. Macrophages and prognosis of oral squamous cell carcinoma: A systematic review. J Oral Pathol Med. 2017. 
1 30. Yamagata Y, Tomioka H, Sakamoto K, et al. CD163-Positive Macrophages Within the Tumor

2 Stroma Are Associated With Lymphangiogenesis and Lymph Node Metastasis in Oral Squamous Cell 3 Carcinoma. J Oral Maxillofac Surg. 2017;75:2144-2153.

4 31. Salo $\mathrm{T}$, Vered $\mathrm{M}$, Bello IO, et al. Insights into the role of components of the tumor 5 microenvironment in oral carcinoma call for new therapeutic approaches. Exp Cell Res. 2014;325:58-64.

6 32. Krishna Priya S, Nagare RP, Sneha VS, et al. Tumour angiogenesis-Origin of blood vessels. Int J 7 Cancer. 2016;139:729-735.

8 33. Subarnbhesaj A, Miyauchi M, Chanbora C, et al. Roles of VEGF-Flt-1 signaling in malignant 9 behaviors of oral squamous cell carcinoma. PLoS One. 2017;12:e187092.

10 34. Song K, Zhu F, Zhang HZ, et al. Tumor necrosis factor-alpha enhanced fusions between oral 11 squamous cell carcinoma cells and endothelial cells via VCAM-1/VLA-4 pathway. Exp Cell Res. $12 \quad 2012 ; 318: 1707-1715$.

13 35. Vered M, Dayan D, Yahalom R, et al. Cancer-associated fibroblasts and epithelial-mesenchymal transition in metastatic oral tongue squamous cell carcinoma. Int J Cancer. 2010;127:1356-1362.

15 36. Bello IO, Vered M, Dayan D, et al. Cancer-associated fibroblasts, a parameter of the tumor 16 microenvironment, overcomes carcinoma-associated parameters in the prognosis of patients with mobile 17 tongue cancer. Oral Oncol. 2011;47:33-38.

18 37. Dourado MR, Guerra ENS, Salo T, et al. Prognostic value of the immunohistochemical detection of cancer-associated fibroblasts in oral cancer: a systematic review and meta-analysis. J Oral Pathol Med. 20 2017.

38. Kelner N, Rodrigues PC, Bufalino A, et al. Activin A immunoexpression as predictor of occult lymph node metastasis and overall survival in oral tongue squamous cell carcinoma. Head Neck. 2015;37:479-486.

39. Sundquist E, Kauppila JH, Veijola J, et al. Tenascin-C and fibronectin expression divide early stage tongue cancer into low- and high-risk groups. Br J Cancer. 2017;116:640-648. 


\section{$1 \quad$ Figure legend}

2 Fig.1: Haematoxylin and eosin staining of an early stage oral tongue squamous cell carcinoma

3 (x100 magnification). A: stroma-poor $(<50 \%)$; B: stroma-rich $(\geq 50 \%)$.

4 Fig. 2: Kaplan-Meier curves for disease-free survival (A) and disease-specific survival (B) of 5 stroma-rich cases compared to stroma-poor in 311 patients with early OTSCC.

6 
Table 1: Relationship between tumour-stroma ratio and age of patient, gender, cTNM stage, histopathologic grade and perineural invasion (PNI)

\begin{tabular}{|c|c|c|c|c|}
\hline \multirow[t]{2}{*}{ Variable } & Total & Stroma-poor & Stroma-rich & $P$ value \\
\hline & $\begin{array}{l}\text { Number (\%) } \\
311\end{array}$ & $\begin{array}{l}\text { Number (\%) } \\
222 \text { (71.4) }\end{array}$ & $\begin{array}{l}\text { Number (\%) } \\
89(28.6)\end{array}$ & \\
\hline Age & & & & 0.07 \\
\hline$\leq 60$ & $129(41.5)$ & $85(38.3)$ & $44(49.4)$ & \\
\hline$>60$ & $182(58.5)$ & $137(61.7)$ & $45(50.6)$ & \\
\hline Gender & & & & 0.90 \\
\hline Male & $165(53)$ & $117(52.7)$ & $48(53.9)$ & \\
\hline Female & $146(47)$ & $105(47.3)$ & $41(46.1)$ & \\
\hline cTNM stage* & 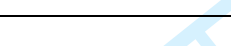 & & & 012 \\
\hline T1N0M0 & $124(39.9)$ & $95(42.8)$ & $29(32.6)$ & \\
\hline T2N0M0 & $187(60.1)$ & $127(57.2)$ & $60(67.4)$ & \\
\hline & & 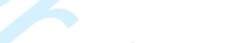 & & \\
\hline Grade (WHO) & & 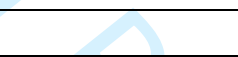 & & 0.39 \\
\hline $\mathrm{I}$ & $105(33.8)$ & $80(36)$ & $25(28.1)$ & \\
\hline II & $131(42.1)$ & $91(41)$ & $40(44.9)$ & \\
\hline III & $75(24.1)$ & $51(23)$ & $24(27)$ & \\
\hline & & +2 & & \\
\hline PNI & & 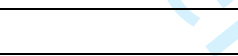 & +2 & 0.04 \\
\hline Absent & $269(86.5)$ & $198(89)$ & $71(79.8)$ & \\
\hline Present & $42(13.5)$ & $24(11)$ & $18(20.2)$ & \\
\hline
\end{tabular}

* cases were staged according to the existing 7th edition of the American Joint Committee on Cancer. 
Table 2: Univariate and multivariate analysis of disease free survival (DFS) and disease specific survival (DSS) for tumour-stroma ratio and other prognostic factors.

\begin{tabular}{|c|c|c|c|c|c|}
\hline \multicolumn{6}{|c|}{ Univariate Analysis } \\
\hline & \multicolumn{2}{|c|}{ DFS } & & \multicolumn{2}{|l|}{ DSS } \\
\hline Variable & HR (95\%CI) & $P$ value & & HR (95\%CI) & $P$ value \\
\hline Age & & 0.011 & & & 0.022 \\
\hline$\leq 60$ & 1 & & & 1 & \\
\hline$>60$ & $1.79(1.14-2.79)$ & & & $1.87(1.09-3.18)$ & \\
\hline Gender & & 0.72 & & & 0.44 \\
\hline Male & 1 & & & 1 & \\
\hline Female & $1.08(0.71-1.64)$ & & & $1.22(0.74-1.99)$ & \\
\hline Stage & & 0.53 & & & 0.15 \\
\hline T1N0M0 & 8 & & & 1 & \\
\hline T2N0M0 & $0.87(0.57-1.33)$ & 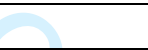 & & $1.48(0.87-2.54)$ & \\
\hline & & 28 & & & \\
\hline Grade & & 0.74 & & & 0.22 \\
\hline $\mathrm{I}$ & 1 & & & 1 & \\
\hline II & $1.11(0.68-1.82)$ & & & $1.68(0.92-3.07)$ & \\
\hline III & $1.25(0.72-2.16)$ & & 1 & $1.58(0.79-3.16)$ & \\
\hline & & & 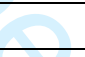 & & \\
\hline PNI & & 0.18 & 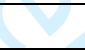 & & 0.49 \\
\hline Absent & 1 & & 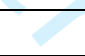 & 1 & \\
\hline Present & $1.46(0.84-2.55)$ & & & $1.27(0.65-2.49)$ & \\
\hline & & & & & \\
\hline HRS & & 0.59 & & 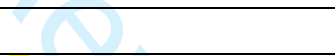 & 0.66 \\
\hline Low-risk & 1 & & & 1 & \\
\hline Intermediate-risk & $0.85(0.36-2.04)$ & & & $1.39(0.42-4.61)$ & \\
\hline High-risk & $0.79(0.34-1.85)$ & & & $1.30(0.40-4.23)$ & \\
\hline & & & & 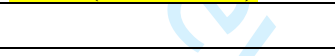 & \\
\hline TSR & & 0.02 & & 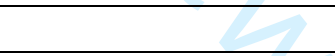 & 0.042 \\
\hline Stroma-poor & 1 & & & 1 & \\
\hline Stroma-rich & $1.67(1.09-2.56)$ & & & $1.69(1.02-2.79)$ & \\
\hline TSR+BD** & & 0.014 & & & $<0.001$ \\
\hline Score 0 & 1 & & & 1 & \\
\hline Score 1 & $1.36(0.74-2.49)$ & & & $5.13(1.78-14.79)$ & \\
\hline Score 2 & $1.73(0.93-3.20)$ & & & $4.99(1.69-14.76)$ & \\
\hline Score 3 & $2.82(1.46-5.42)$ & & & $10.43(3.51-31.01)$ & \\
\hline \multicolumn{6}{|c|}{ Multivariate Analysis } \\
\hline Age & & 0.003 & & & 0.007 \\
\hline$\leq 60$ & 1 & & & 1 & \\
\hline$>60$ & $2.02(1.27-3.23)$ & & & $2.17(1.24-3.79)$ & \\
\hline TSR & & 0.008 & & & 0.03 \\
\hline Stroma-poor & 1 & & & 1 & \\
\hline
\end{tabular}




\begin{tabular}{|l|l|l|l|l|l|}
\hline Stroma-rich & $1.81(1.17-2.79)$ & & & $1.71(1.02-2.86)$ & \\
\hline & & & & & \\
\hline TSR+BD $* *$ & & 0.004 & & & $<0.001$ \\
\hline Score 0 & 1 & & & 1 & \\
\hline Score 1 & $1.48(0.79-2.77)$ & & & $5.37(1.85-15.63)$ & \\
\hline Score 2 & $1.74(0.93-3.26)$ & & & $4.90(1.65-14.57)$ & \\
\hline Score 3 & $3.42(1.71-6.82)$ & & & $11.63(3.83-35.31)$ & \\
\hline
\end{tabular}

**The scores of TSR were combined with our previous tumour budding and depth of invasion (BD) model as explained in the Methods section.

\section{Abbreviations:}

PNI: Perineural invasion; HRS: Histologic risk score; TSR: Tumour-stroma ratio. 

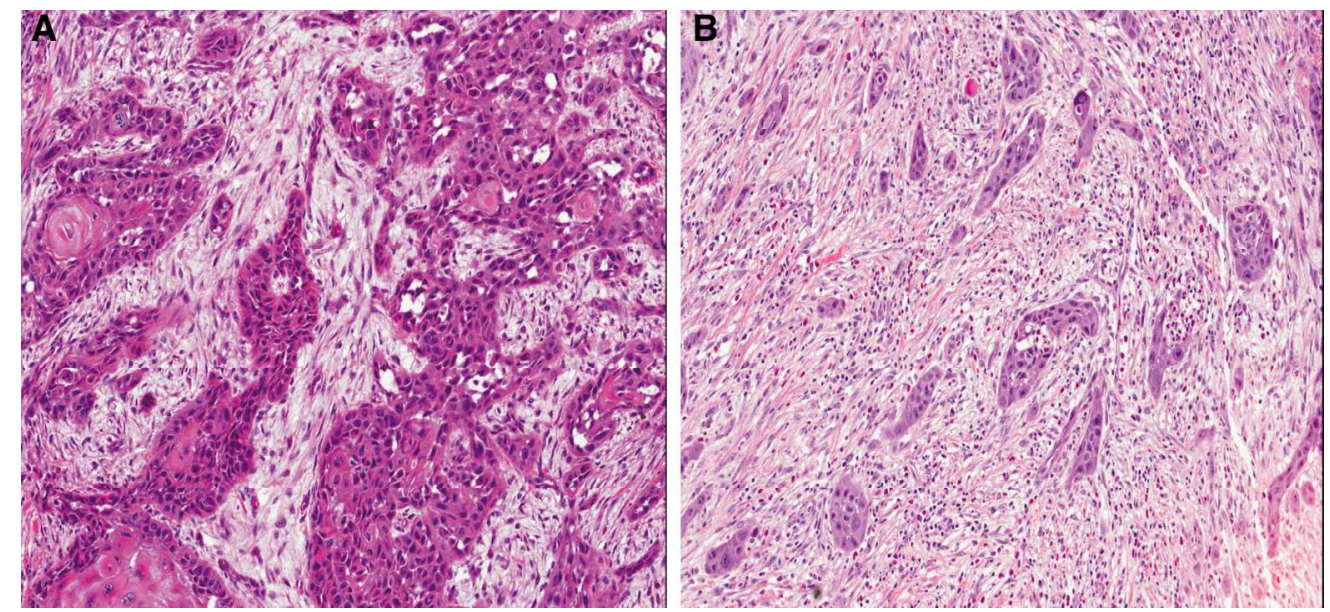

Figure 1

$135 \times 62 \mathrm{~mm}(300 \times 300 \mathrm{DPI})$

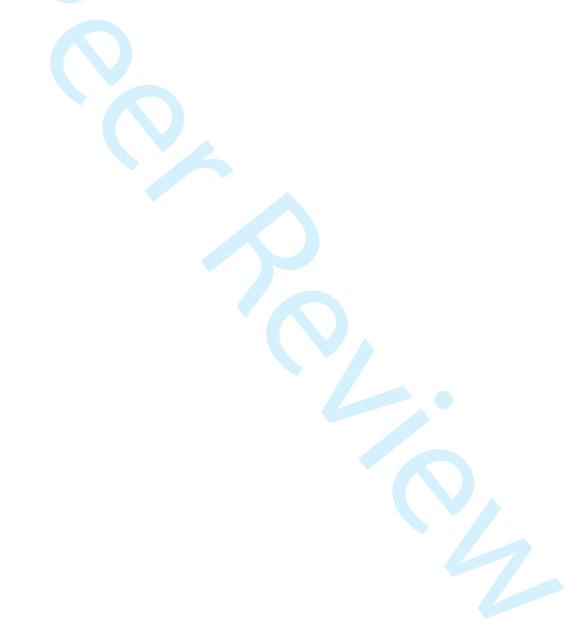



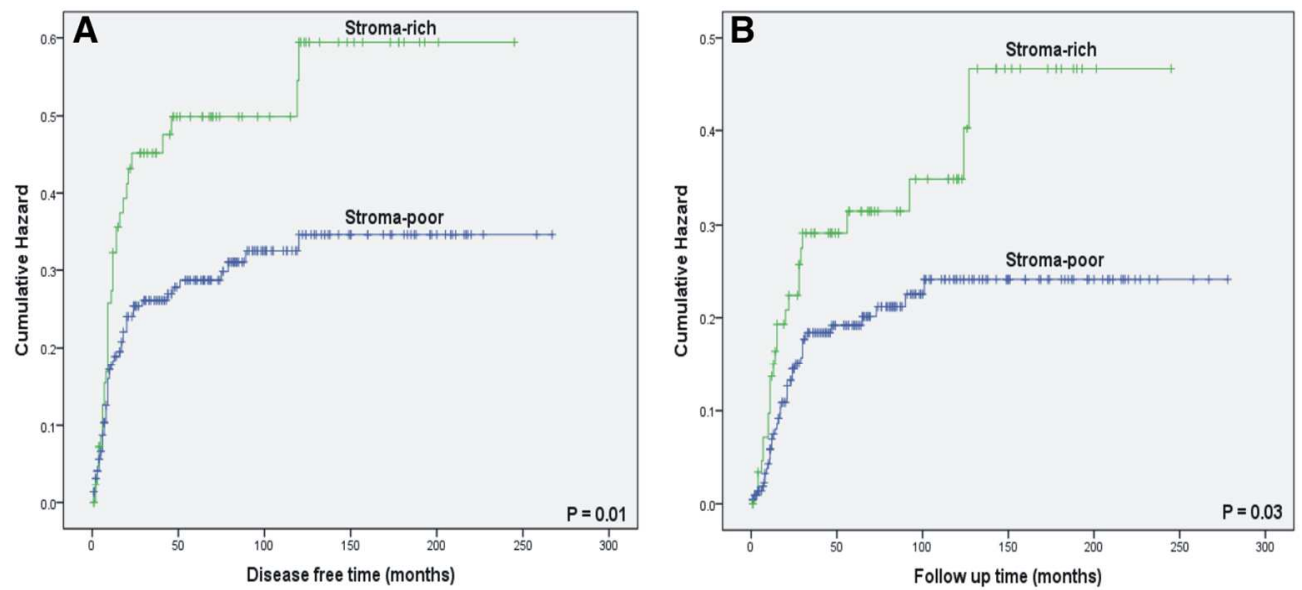

Figure 2

$135 \times 62 \mathrm{~mm}(300 \times 300$ DPI $)$ 